\title{
TORSION MATRICES OVER COMMUTATIVE INTEGRAL GROUP RINGS
}

\author{
Gregory T. Lee and Sudarshan K. Sehgal
}

Abstract

Let $\mathbb{Z} A$ be the integral group ring of a finite abelian group $A$, and $n$ a positive integer greater than 5 . We provide conditions on $n$ and $A$ under which every torsion matrix $U$, with identity augmentation, in $G L_{n}(\mathbb{Z} A)$ is conjugate in $G L_{n}(\mathbb{Q} A)$ to a diagonal matrix with group elements on the diagonal. When $A$ is infinite, we show that under similar conditions, $U$ has a group trace and is stably conjugate to such a diagonal matrix.

Let $\mathbb{Z} G$ be the integral group ring of a finite group, $G$. Let $\epsilon: \mathbb{Z} G \rightarrow$ $\mathbb{Z}$ be the augmentation map, defined by $\epsilon\left(\sum_{g \in G} \alpha_{g} g\right)=\sum_{g \in G} \alpha_{g}$. A conjecture due to Zassenhaus is

(ZC1). If $u \in \mathbb{Z} G, u^{n}=1$, and $\epsilon(u)=1$, then there exists a unit $\alpha \in$ $\mathbb{Q} G$ such that $\alpha^{-1} u \alpha \in G$.

This is known to be true for finite nilpotent groups (see [9, Theorem 40.4]), but remains open for finite groups in general.

In some cases, it is possible to translate the problem into a question about matrices, which is interesting in its own right. (See, for instance, $[5]$.) For a group $G$ and a positive integer $n$, we define $\epsilon^{*}: G L_{n}(\mathbb{Z} G) \rightarrow$ $G L_{n}(\mathbb{Z})$ by applying $\epsilon$ to each matrix entry. Let $S G L_{n}(\mathbb{Z} G)=\operatorname{ker}\left(\epsilon^{*}\right)$. That is, $S G L_{n}(\mathbb{Z} G)$ is the group of invertible $n \times n$ matrices over $\mathbb{Z} G$ with identity augmentation. We have this

Problem. For a finite abelian group $A$ and a positive integer $n$, is it true that for every torsion matrix $U \in S G L_{n}(\mathbb{Z} A), U$ is conjugate in $G L_{n}(\mathbb{Q} A)$ to a diagonal matrix with group elements on the diagonal?

2000 Mathematics Subject Classification. Primary: 20H25, 16S34.

The first author was supported by an Izaak Walton Killam Memorial Scholarship.

The second author was supported by NSERC of Canada. 
For $n=1$, a positive answer for all $A$ is classical (see $[\mathbf{9}$, Corollary 1.6]). Luthar and Passi obtained a positive answer for all $A$ when $n=2$, if $\mathbb{Q} A$ is replaced with $\mathbb{C} A$, in [4]. More recently, in [8], Marciniak and Sehgal obtained an affirmative answer for all $A$ and all $n \leq 5$. On the other hand, in [3], Cliff and Weiss constructed a counterexample for the case $n=6, A=C_{6} \times C_{6}$. Furthermore, they showed that for a given finite abelian (or, indeed, nilpotent) group $A$, the answer will be yes for all $n$ if and only if $A$ has at most one non-cyclic Sylow subgroup.

Our question, then, is this: if $A$ has two or more non-cyclic Sylow subgroups, by restricting them suitably, can we obtain a positive answer for certain $n \geq 6$ ? We have this result.

Theorem 1. Let $A$ be a finite abelian group and $n \geq 6$. Suppose that either

(1) A has at most one non-cyclic Sylow subgroup; or,

(2) if $q_{1}$ and $q_{2}$ are the two smallest (distinct) primes such that the Sylow $q_{1}$ - and $q_{2}$-subgroups of $A$ are non-cyclic, then $q_{1}+q_{2}>$ $\frac{n^{2}+n-8}{4}$.

Then for any torsion matrix $U \in S G L_{n}(\mathbb{Z} A), U$ is conjugate in $G L_{n}(\mathbb{Q} A)$ to a diagonal matrix with group elements on the diagonal.

We will follow the same plan of attack as in [8]. For any matrix $M$, let $\operatorname{Tr}(M)$ denote its trace. Also, if $\alpha \in \mathbb{Z} A$, we say that $\alpha \geq 0$ if every coefficient of $\alpha$ is greater than or equal to zero.

By the proposition in $[\mathbf{8}]$, the theorem is equivalent to showing that $\operatorname{Tr}(U) \geq 0$. Suppose that our theorem fails. Fix $n \geq 6$, and choose an abelian group $A$ of minimal order which provides us with a counterexample $U$. Note that if $A$ satisfies (1), then [3] tells us that our result holds, hence we may assume that (2) is satisfied. Let us write $\operatorname{Tr}(U)=\alpha \in \mathbb{Z} A$, where $\alpha \geq 0$. We also write $\alpha=\alpha_{+}-\alpha_{-}$, where $\alpha_{+}, \alpha_{-} \geq 0, S_{+}=\operatorname{supp} \alpha_{+}, S_{-}=\operatorname{supp} \alpha_{-}$, and $S_{+}$and $S_{-}$are disjoint. If $h \in S_{-}$, we may replace $U$ with $h^{-1} U$, and therefore assume that $1 \in S_{-}$. More explicitly, we write $\alpha_{+}=\sum_{g \in S_{+}} \alpha_{g} g, \alpha_{-}=\sum_{g \in S_{-}} \alpha_{g} g$. We have

Lemma 1. (a) $\sum_{g \in S_{+}} \alpha_{g}-\sum_{g \in S_{-}} \alpha_{g}=n$, and (b) $\sum_{g \in A} \alpha_{g}^{2}<n^{2}$.

Proof: (a) The expression $\sum_{g \in S_{+}} \alpha_{g}-\sum_{g \in S_{-}} \alpha_{g}$ is the trace of the augmentation of $U$, namely the trace of the identity matrix. (b) $[\mathbf{4}$, Corollary 2.3].

Lemma 2. $\left|S_{+}\right| \leq \frac{n^{2}+n}{2}-1$. 
Proof: Suppose this is not the case. Then $\epsilon\left(\alpha_{+}\right) \geq\left|S_{+}\right| \geq \frac{n^{2}+n}{2}$ (since the coefficients of $\alpha_{+}$and $\alpha_{-}$are positive). By Lemma 1, $\epsilon\left(\alpha_{-}\right)=$ $\epsilon\left(\alpha_{+}\right)-n \geq \frac{n^{2}-n}{2}$. Thus, by Lemma 1 ,

$$
n^{2}>\sum_{g \in A} \alpha_{g}^{2} \geq \sum_{g \in S_{+}} \alpha_{g}+\sum_{g \in S_{-}} \alpha_{g} \geq \frac{n^{2}+n}{2}+\frac{n^{2}-n}{2}=n^{2}
$$

which is a contradiction.

For each prime $p$, let $\mathcal{E}_{p}$ be the set of all subgroups of order $p$ in $A$. Let $\mathcal{E}=\bigcup_{p} \mathcal{E}_{p}$. We define $\sigma$ to be $|\mathcal{E}|$. By assumption, we know that $A$ contains a copy of $C_{q_{1}} \times C_{q_{1}} \times C_{q_{2}} \times C_{q_{2}}$. Therefore, $\sigma \geq q_{1}+q_{2}+2>\frac{n^{2}+n}{4}$. For any $x \in S_{-}$and any $H \in \mathcal{E}$, we let $T_{x, H}=H x \cap S_{+}$. We will prove

Lemma 3. No $T_{x, H}$ is empty.

Proof: We have the usual projection $\pi: \mathbb{Z} A \rightarrow \mathbb{Z}(A / H)$. Applying this to each element in the matrix, we see that $\pi(U)$ is a torsion element in $S G L_{n}(\mathbb{Z}(A / H))$. Now, looking at the restrictions placed upon $A$ in the theorem, we observe that any homomorphic image of an abelian group satisfying (1) will also satisfy (1), and the homomorphic image of an abelian group satisfying (2) must satisfy (1) or (2). Thus, $A / H$ is also a group of the type discussed in the theorem. Since $A$ is a group of minimal order which provides a counterexample, $\pi(\alpha)=\operatorname{Tr}(\pi(U)) \geq 0$. Now, $\pi(x)$ appears in the support of $\pi\left(\alpha_{-}\right)$(since all coefficients are positive). Thus, $\pi(x)$ must also appear in the support of $\pi\left(\alpha_{+}\right)$. That is, $T_{x, H}=S_{+} \cap \pi^{-1}(\pi(x))$ is nonempty.

\section{Lemma 4.}

(i) For any $H \in \mathcal{E}$ and $x, y \in S_{-}$, the sets $T_{x, H}$ and $T_{y, H}$ are either disjoint or identical. In fact, they coincide if and only if $x y^{-1} \in$ $H$.

(ii) Assume $H, K \in \mathcal{E}$, with $H \neq K$. For any $x, y \in S_{-}$, if $T_{x, H} \cap T_{y, K}$ is nonempty then $x y^{-1} \in H K \backslash(H \cup K)$ and $\left|T_{x, H} \cap T_{y, K}\right|=1$.

Proof: See [8, Lemma 4].

For each $x \in S_{-}$, we define $T_{x}=\bigcup_{H \in \mathcal{E}} T_{x, H}$. It is important to notice that $T_{x}$ is a subset of $S_{+}$which is a disjoint union of $\sigma$ sets (by the last lemma, since $x x^{-1}=1 \in H \cup K$ for any $\left.H \neq K\right)$. Hence $\left|T_{x}\right| \geq \sigma>\frac{n^{2}+n}{4}$. Let us examine the intersections of the sets $T_{x}$. 
Lemma 5. Let $x$ and $y$ be distinct elements of $S_{-}$. If $T_{x} \cap T_{y}$ is nonempty, then either

(i) $x y^{-1}$ has order $p q$ for distinct primes $p$ and $q$, and then $\left|T_{x} \cap T_{y}\right| \leq$ 2 ; or

(ii) $x y^{-1}$ is a p-element for some prime $p$, and

$$
T_{x} \cap T_{y} \subseteq \bigcup_{H, K \in \mathcal{E}_{p}} T_{x, H} \cap T_{y, K} .
$$

Proof: If $T_{x} \cap T_{y}$ is not empty, then we may choose $H \in \mathcal{E}_{p}, K \in \mathcal{E}_{q}$, for (not necessarily distinct) primes $p$ and $q$, such that $T_{x, H} \cap T_{y, K}$ is not empty. First, suppose $p \neq q$. By Lemma $4, x y^{-1} \in H K \backslash(H \cup K)$. But this set is precisely the set of elements of order $p q$ in $H K$, hence $x y^{-1}$ has order $p q$. Also, $H$ and $K$ are uniquely determined as the Sylow subgroups of $H K=\left\langle x y^{-1}\right\rangle$. That is, if $\left\{H^{\prime}, K^{\prime}\right\} \neq\{H, K\}$, then $T_{x, H^{\prime}} \cap T_{y, K^{\prime}}$ is empty. Therefore,

$$
\left|T_{x} \cap T_{y}\right|=\left|\left(T_{x, H} \cap T_{y, K}\right) \cup\left(T_{x, K} \cap T_{y, H}\right)\right| \leq 2,
$$

by Lemma 4 .

If $p=q$, then by Lemma $4, x y^{-1} \in H K$, hence it is a $p$-element. It follows easily that $T_{x, H^{\prime}} \cap T_{y, K^{\prime}}$ must be empty unless $H^{\prime}$ and $K^{\prime}$ are -groups.

For the proof of the next lemma, we refer to the proof of [8, Lemma 6], noting only that a homomorphic image of a group satisfying (1) or (2) must also satisfy (1) or (2).

Lemma 6. $\epsilon\left(\alpha_{+}\right) \geq \sigma \cdot \max \left\{\alpha_{g}: g \in S_{-}\right\}$.

Lemma 7. $\alpha_{-}=\sum_{g \in S_{-}} g$. In particular, $\epsilon\left(\alpha_{-}\right)=\left|S_{-}\right|$.

Proof: Suppose that not all coefficients of $\alpha_{-}$are 1. Then by Lemma 6 , $\epsilon\left(\alpha_{+}\right) \geq 2 \sigma>\frac{n^{2}+n}{2}$. By Lemma $1, \epsilon\left(\alpha_{-}\right)=\epsilon\left(\alpha_{+}\right)-n>\frac{n^{2}-n}{2}$. Just as in the proof of Lemma 2, this is a contradiction.

Lemma 8. $\left|S_{-}\right|>\frac{n^{2}-3 n}{4}$.

Proof: By Lemma $6, \epsilon\left(\alpha_{+}\right) \geq \sigma>\frac{n^{2}+n}{4}$. Then $\left|S_{-}\right|=\epsilon\left(\alpha_{-}\right)=\epsilon\left(\alpha_{+}\right)-$ $n>\frac{n^{2}-3 n}{4}$.

Clearly, since $n \geq 6$, this means $\left|S_{-}\right|>4$. For any distinct $x, y \in S_{-}$, we say that $T_{x}$ and $T_{y}$ have a large intersection if $x y^{-1}$ is a $p$-element. Otherwise, the intersection is said to be small. (By Lemma 5, the intersection can contain at most two elements in this case.) Our last lemma is 
Lemma 9. There exist distinct elements $x$ and $y$ in $S_{-}$such that $T_{x}$ and $T_{y}$ have small intersection.

Proof: The proof of [8, Lemma 9] carries through verbatim up to the point where they conclude that $\left|S_{+}\right| \geq \sigma+\left|S_{-}\right|-1$. From this, we deduce that

$n=\epsilon\left(\alpha_{+}\right)-\left|S_{-}\right| \geq\left|S_{+}\right|-\left|S_{-}\right| \geq \sigma+\left|S_{-}\right|-1-\left|S_{-}\right|>\frac{n^{2}+n}{4}-1>n$ for $n \geq 6$. This is a contradiction.

Proof of Theorem 1: We claim that $\left|S_{+}\right| \geq 2 \sigma-1$. This would complete the proof, since $\sigma>\frac{n^{2}+n}{4}$, hence $\left|S_{+}\right|>\frac{n^{2}+n}{2}-1$, and this contradicts Lemma 2. Let us prove the claim.

We know that each $\left|T_{u}\right| \geq \sigma$. If any two such sets are disjoint, then $\left|S_{+}\right| \geq 2 \sigma$, and we are done. Thus, we will assume that no two $T_{u}$ 's are disjoint. Suppose that for some pairwise distinct $x, y, z \in S_{-}, T_{x}$ and $T_{y}$ have small intersection, and $T_{y}$ and $T_{z}$ have small intersection. We have two cases. First, if $T_{x}$ and $T_{z}$ have small intersection, then

$$
\begin{aligned}
&\left|T_{x} \cup T_{y} \cup T_{z}\right|=\left|T_{x}\right|+\left|T_{y} \backslash\left(T_{x} \cap T_{y}\right)\right|+\left|T_{z} \backslash\left(\left(T_{x} \cup T_{y}\right) \cap T_{z}\right)\right| \\
& \geq \sigma+(\sigma-2)+(\sigma-4)
\end{aligned}
$$

since each set has order at least $\sigma$, and any pair has at most two elements in common. But then $\left|S_{+}\right| \geq 3 \sigma-6>2 \sigma-1$, since $\sigma>\frac{n^{2}+n}{4}>10$. Second, if $T_{x}$ and $T_{z}$ have large intersection, then by Lemma 5 , there exists a prime $p$ such that $T_{x} \cap T_{z} \subseteq \bigcup_{H, K \in \mathcal{E}_{p}} T_{x, H} \cap T_{z, K} \subseteq \bigcup_{H \in \mathcal{E}_{p}} T_{x, H}$. Choose one of $\left\{q_{1}, q_{2}\right\}$ which is not $p$ (without loss of generality, say $q_{1}$ ). Then $T_{x} \backslash\left(T_{x} \cap T_{z}\right) \supseteq \bigcup_{H \in \mathcal{E}_{q_{1}}} T_{x, H}$, since the $T_{x, H}$ are disjoint, for a fixed $x$, by Lemma 4. Again, we have

$$
\left|T_{x} \cup T_{y} \cup T_{z}\right|=\left|T_{y}\right|+\left|T_{z} \backslash\left(T_{z} \cap T_{y}\right)\right|+\left|T_{x} \backslash\left(T_{x} \cap\left(T_{y} \cup T_{z}\right)\right)\right| .
$$

Now, $\left|T_{y}\right| \geq \sigma$, and since $T_{z}$ and $T_{y}$ have small intersection, $\mid T_{z} \backslash\left(T_{z} \cap\right.$ $\left.T_{y}\right) \mid \geq \sigma-2$. Also, $\left|T_{x} \backslash\left(T_{x} \cap T_{z}\right)\right| \geq\left|\bigcup_{H \in \mathcal{E}_{q_{1}}} T_{x, H}\right| \geq q_{1}+1$, since the $T_{x, H}$ are nonempty, disjoint, and there are at least $q_{1}+1$ of them, by choice of $q_{1}$. Now, $\left|T_{x} \cap T_{y}\right| \leq 2$, hence $\left|T_{x} \backslash\left(T_{x} \cap\left(T_{y} \cup T_{z}\right)\right)\right| \geq q_{1}+1-2=$ $q_{1}-1$. Thus,

$$
\left|T_{x} \cup T_{y} \cup T_{z}\right| \geq \sigma+\sigma-2+q_{1}-1 \geq 2 \sigma-1,
$$

since $q_{1}$, being a prime, is at least 2 . This is what we wanted to know, and therefore

(*) We may assume that, for any distinct $a, b, c \in S_{-}$, either $T_{a}$ and $T_{b}$ have large intersection, or $T_{b}$ and $T_{c}$ have large intersection. 
We know from Lemma 9 that there exist distinct $x$ and $z$ in $S_{-}$such that $T_{x}$ and $T_{z}$ have small intersection. Since they cannot be disjoint, Lemma 5 tells us that $x z^{-1}$ has order $p q$ for distinct primes $p$ and $q$. We know from Lemma 8 that $\left|S_{-}\right| \geq 5$, so let us say that $v, w, x$, $y$ and $z$ are distinct elements of $S_{-}$. By $(*), T_{x}$ and $T_{y}$ cannot have small intersection hence, by Lemma $5, x y^{-1}$ is an $r$-element for some prime $r$. If $p \neq r \neq q$, then $y z^{-1}=\left(x y^{-1}\right)^{-1} x z^{-1}$ has order divisible by three primes, contradicting Lemma 5 . Thus, $x y^{-1}$ is a $p$-element or a $q$-element. Without loss of generality, it is a $p$-element. Then $y z^{-1}$, being the product of an element of order $p q$ and a $p$-element, must have order $q$ or $p q$ (given the choices afforded by Lemma 5). In the latter case, $T_{y}$ and $T_{z}$ have small intersection, which is disallowed by $(*)$, hence $y z^{-1}$ is a $q$-element. Again by $(*), T_{x}$ and $T_{w}$ have large intersection, hence $x w^{-1}$ is an $r$-element for some prime $r$. If $p \neq r \neq q$, then $z w^{-1}=\left(x z^{-1}\right)^{-1} x w^{-1}$ has order divisible by $p, q$, and $r$, which is impossible. Thus, $x w^{-1}$ is a $p$-element or a $q$-element. Suppose $x w^{-1}$ is a $p$-element. Then since $x y^{-1}$ is a $p$-element, so is $w y^{-1}=\left(x w^{-1}\right)^{-1} x y^{-1}$. Now, $z w^{-1}=\left(x z^{-1}\right)^{-1} x w^{-1}$. Since $x z^{-1}$ has order $p q$ and $x w^{-1}$ is a $p$-element, $z w^{-1}$ must have order $q$ or $p q$. Once again, $(*)$ disallows the latter, hence $z w^{-1}$ is a $q$-element. But $w y^{-1}=\left(z w^{-1}\right)^{-1}\left(y z^{-1}\right)^{-1}$, and both $z w^{-1}$ and $y z^{-1}$ are $q$-elements. Therefore, $w y^{-1}$ is both a $p$-element and a $q$-element, which is impossible. It follows that $x w^{-1}$ must be a $q$-element. Thus, $w y^{-1}=\left(x w^{-1}\right)^{-1} x y^{-1}$, being the product of a $q$-element and a $p$-element, has order $p q$.

Once again, $T_{x}$ and $T_{v}$ must have large intersection. Thus, $x v^{-1}$ is an $r$-element for some prime $r$, and once again, $r=p$ or $q$. Suppose $x v^{-1}$ is a $p$-element. Then $y v^{-1}=\left(x y^{-1}\right)^{-1} x v^{-1}$, being a product of two $p$-elements, is a $p$-element. However, $z v^{-1}=\left(x z^{-1}\right)^{-1} x v^{-1}$. Since $x z^{-1}$ has order $p q$, and $x v^{-1}$ is a $p$-element, we again see that $z v^{-1}$ is a $q$-element. But $y z^{-1}$ is also a $q$-element, hence $y v^{-1}=y z^{-1} z v^{-1}$ is both a $p$-element and a $q$-element, giving us a contradiction. Therefore, $x v^{-1}$ is a $q$-element. But then $y v^{-1}=\left(x y^{-1}\right)^{-1} x v^{-1}$ is the product of a $p$-element and a $q$-element, hence it has order $p q$. That is, $T_{y}$ and $T_{v}$ have small intersection, but $T_{w}$ and $T_{y}$ also have small intersection, and this contradicts $(*)$. The proof is complete.

Of course, the restriction placed upon the group becomes much harsher as $n$ increases, but for small values of $n$, it is fairly mild. For instance, if $n=6$, we are assuming that $q_{1}+q_{2} \geq 9$. In this case, the theorem reduces to this 
Corollary. Let $A$ be a finite abelian group. Suppose that at most one of the Sylow p-subgroups, $p \leq 5$, is non-cyclic. Then for any torsion matrix $U \in S G L_{6}(\mathbb{Z} A), U$ is conjugate in $G L_{6}(\mathbb{Q} A)$ to a diagonal matrix with group elements on the diagonal.

This improves [5, Theorem 4.6] which requires us to assume that $n<p$ for all primes $p$ dividing the order of $A$.

Removing the assumption that $A$ is finite, we would also like to know when a torsion matrix $U \in S G L_{n}(\mathbb{Z} A)$ will have a group trace. A matrix $U \in G L_{n}(\mathbb{Z} A)$ is said to have a group trace if there exist $g_{1}, \ldots, g_{n} \in A$ such that, for all positive integers $m, \operatorname{Tr}\left(U^{m}\right)=\sum_{i=1}^{n} g_{i}^{m}$. (Note that this definition applies only to abelian groups.) See [1] and [2] for a more extensive discussion of this property. In [2, Theorem 3.3], it is shown that if $n<p$ for every prime $p$ such that $A$ has $p$-torsion, then every torsion matrix $U \in S G L_{n}(\mathbb{Z} A)$ will have a group trace. In a similar vein, we can prove

Theorem 2. Let $A$ be an abelian group and $n$ a positive integer. Suppose either that $n \leq 5$ or we have

(1) every finite subgroup of $A$ has at most one non-cyclic Sylow subgroup; or,

(2) if $q_{1}$ and $q_{2}$ are the two smallest (distinct) primes such that the Sylow $q_{1}$ - and $q_{2}$-subgroups of some finite subgroup of $A$ are noncyclic, then $q_{1}+q_{2}>\frac{n^{2}+n-8}{4}$.

Then every torsion matrix $U \in S G L_{n}(\mathbb{Z} A)$ has a group trace.

Proof: Since the condition on $A$ is certainly inherited by subgroups, there is no harm in assuming that $A$ is generated by the group elements appearing in the support of one or more entries of $U$. In particular, $A$ is finitely generated. In [2, pp. 629-630] it is shown that in this case, the elements of infinite order in $A$ do not appear in the support of $\operatorname{Tr}\left(U^{r}\right)$ for any $r \geq 1$. Let us write $A=T \times F$, where $T$ is finite and $F$ is a free abelian group. Then the support of $\operatorname{Tr}\left(U^{r}\right)$ is contained in $T$ for all $r \geq 1$. It follows from [1, Proposition 15] that if the image of $U$ in $S G L_{n}(\mathbb{Z}(A / F))=S G L_{n}(\mathbb{Z} T)$ has a group trace, then $U$ has a group trace. In effect, we have reduced the problem to the case in which $A$ is finite. But by the main result of [8] (if $n \leq 5$ ) or Theorem 1 (if $n \geq 6$ ), $U$ is conjugate to a diagonal matrix $\operatorname{diag}\left(g_{1}, \ldots, g_{n}\right)$ in this case. It follows immediately that $U$ has a group trace. 
Since we are dealing with abelian groups, the restriction on the Sylow subgroups of finite subgroups of $A$ could be replaced with a restriction on the Sylow subgroups of $A$. However, requiring such subgroups to be cyclic is too strong a condition. For example, there is no reason to rule out groups which are the direct product of quasicyclic $p$-groups, $\mathbb{Z}_{p^{\infty}}$ for different primes $p$.

When dealing with infinite groups, it would be rather optimistic to expect our matrix $U$ to be conjugate to a diagonal matrix, particularly since even (ZC1) fails for infinite nilpotent groups (see [7]). Instead, let us introduce the following notion. Let $K$ be a subfield of the complex numbers and $G$ a group. For any positive integer $n$, we say that two matrices $A, B \in G L_{n}(K G)$ are stably conjugate if there exist roots of unity $\xi_{1}, \ldots, \xi_{k} \in K$ such that

$$
\left(\begin{array}{cccc}
A & 0 & \cdots & 0 \\
0 & \xi_{1} & & 0 \\
\vdots & & \ddots & \\
0 & 0 & & \xi_{k}
\end{array}\right) \text { and }\left(\begin{array}{cccc}
B & 0 & \cdots & 0 \\
0 & \xi_{1} & & 0 \\
\vdots & & \ddots & \\
0 & 0 & & \xi_{k}
\end{array}\right)
$$

are conjugate in $G L_{n+k}(K G)$. For the definition of the Bass rank map, to which we will refer in the proof below, we refer the reader to $[\mathbf{6}$, p. 572]. Let $\overline{\mathbb{Q}}$ denote the algebraic closure of $\mathbb{Q}$ in $\mathbb{C}$. We have

Theorem 3. Let $A$ be an abelian group and $n$ a positive integer. Suppose either that $n \leq 5$ or else (1) or (2) of Theorem 2 holds. Then every torsion matrix $U \in S G L_{n}(\mathbb{Z} A)$, regarded as a matrix in $G L_{n}(\overline{\mathbb{Q}} A)$, is stably conjugate to a diagonal matrix with group elements on the diagonal.

Proof: Once again, we are free to assume that $A$ is finitely generated. Let us write $A=T \times F$, where $T$ is finite and $F$ is a free abelian group. By [6, Theorem 4.1], if $K$ is a splitting field for $T$ in $\mathbb{C}$, then the Bass rank map is injective on $K_{0}(K A)$. By Brauer's Theorem, this only requires $K$ to contain a primitive $e$-th root of unity, where $e$ is the exponent of $T$. Let $m=d e$, where $d$ is the multiplicative order of $U$. Then, let us take $K=\mathbb{Q}(\xi)$, where $\xi$ is a primitive $m$-th root of unity. By [1, Proposition 14], $U$ is stably conjugate over $K A$ to a diagonal matrix with group elements on the diagonal if and only if $U$ has a group trace. By Theorem 2, $U$ does indeed have a group trace. Enlarging the field to $\overline{\mathbb{Q}}$ does not harm our conclusion. Therefore, we are done.

Remark. The definition of stable conjugacy in [1] is slightly different from the one we have used. In that paper, the scalars $\xi_{i}$ were not assumed to be roots of unity. However, examining the relevant proofs (to wit, the 
proofs of Propositions 13 and 14), we can see that only roots of unity were used. In addition, in view of [2, Theorem 3.3], it will also suffice to assume in Theorem 3 that $n<p$ for every prime $p$ such that $A$ has p-torsion.

\section{References}

[1] A. Bovdi, Z. MarciniaK and S. K. Sehgal, Torsion units in infinite group rings, J. Number Theory 47 (1994), 284-299.

[2] G. K. Chadha And I. B. S. Passi, Bass conjecture and the group trace property, Comm. Algebra 26 (1998), 627-639.

[3] G. Cliff AND A. WeIss, Finite groups of matrices over group rings, Trans. Amer. Math. Soc. 352 (2000), 457-475.

[4] I. S. Luthar And I. B. S. PASsi, Torsion units in matrix group rings, Comm. Algebra 20 (1992), 1223-1228.

[5] Z. Marciniak, J. Ritter, S. K. Sehgal and A. Weiss, Torsion units in integral group rings of some metabelian groups. II, J. Number Theory 25 (1987), 340-352.

[6] Z. S. Marciniak and S. K. Sehgal, Finite matrix groups over nilpotent group rings, J. Algebra 181 (1996), 565-583.

[7] Z. S. Marciniak and S. K. Sehgal, Zassenhaus conjecture and infinite nilpotent groups, J. Algebra 184 (1996), 207-212.

[8] Z. S. MarciniaK and S. K. Sehgal, Torsion matrices over abelian group rings, J. Group Theory 3 (2000), 67-75.

[9] S. K. SeHGaL, "Units in integral group rings", Longman, New York, 1993.

Department of Mathematical Sciences

University of Alberta

Edmonton

Alberta, Canada T6G 2G1

E-mail address: glee@vega.math.ualberta.ca

E-mail address: s.sehgal@ualberta.ca

Current address of Gregory T. Lee:

Department of Mathematics

University of Wisconsin-Madison

Madison, Wisconsin 53706

U.S.A.

E-mail address: glee@math.wisc.edu

Primera versió rebuda el 7 de gener de 1999, darrera versió rebuda el 13 de juny de 2000. 